\title{
Systematic Approach to Developing Splice Modulating Antisense Oligonucleotides
}

\author{
May T. Aung-Htut ${ }^{1,2,+}\left(\mathbb{D}\right.$, Craig S. McIntosh ${ }^{1,2,+} \oplus$, Kristin A. Ham ${ }^{1}$, Ianthe L. Pitout ${ }^{1,2}$, \\ Loren L. Flynn ${ }^{1,2}$, Kane Greer ${ }^{1}$, Sue Fletcher ${ }^{1,2}$ and Steve D. Wilton ${ }^{1,2, *}$ \\ 1 Centre for Molecular Medicine and Innovative Therapeutics, Murdoch University, Perth, WA 6150, Australia; \\ M.Aung-Htut@murdoch.edu.au (M.T.A-H.); C.McIntosh@murdoch.edu.au (C.S.M.); \\ Kristin.Ham@murdoch.edu.au (K.A.H.); I.pitout@murdoch.edu.au (I.L.P.); \\ Loren.Flynn@murdoch.edu.au (L.L.F.); k.greer@murdoch.edu.au (K.G.); s.fletcher@murdoch.edu.au (S.F.) \\ 2 Perron Institute for Neurological and Translational Science, Centre for Neuromuscular and Neurological \\ Disorders, The University of Western Australia, Perth, WA 6009, Australia \\ * Correspondence: s.wilton@murdoch.edu.au \\ + These authors contributed equally to this work.
}

Received: 27 September 2019; Accepted: 10 October 2019; Published: 11 October 2019

\begin{abstract}
The process of pre-mRNA splicing is a common and fundamental step in the expression of most human genes. Alternative splicing, whereby different splice motifs and sites are recognised in a developmental and/or tissue-specific manner, contributes to genetic plasticity and diversity of gene expression. Redirecting pre-mRNA processing of various genes has now been validated as a viable clinical therapeutic strategy, providing treatments for Duchenne muscular dystrophy (inducing specific exon skipping) and spinal muscular atrophy (promoting exon retention). We have designed and evaluated over 5000 different antisense oligonucleotides to alter splicing of a variety of pre-mRNAs, from the longest known human pre-mRNA to shorter, exon-dense primary gene transcripts. Here, we present our guidelines for designing, evaluating and optimising splice switching antisense oligomers in vitro. These systematic approaches assess several critical factors such as the selection of target splicing motifs, choice of cells, various delivery reagents and crucial aspects of validating assays for the screening of antisense oligonucleotides composed of 2'-O-methyl modified bases on a phosphorothioate backbone.
\end{abstract}

Keywords: antisense oligonucleotide; splice modulation; 2'-O-Methyl; transfection

\section{Introduction}

Splice switching antisense oligonucleotides (AOs) are gaining interest as therapeutics for a wide variety of inherited and acquired diseases, with the approvals of Eteplirsen and Nusinersen to treat Duchenne muscular dystrophy and spinal muscular atrophy, respectively [1-3]. Antisense oligonucleotides are short, synthetic nucleic acids or analogues, typically 18 to 30 nucleotides in length that can specifically anneal to a complementary DNA or RNA sequence via Watson-Crick base-pairing [4]. Depending upon the nature of the AO (modified bases and backbone chemistry), these compounds can be designed to alter gene expression through several distinct mechanisms; including, but not limited to, RNase H mediated mRNA degradation [5], induction of RNA silencing [6], translation blockade [7], suppression of miRNA action, and modulation of pre-mRNA splicing [8]. The specific mechanism induced is determined by the AO chemistry as well as the annealing location in the gene transcript. Here, we specifically focus on AO design and delivery to modulate pre-mRNA processing, by redirecting splice site/motif selection to promote either specific exon skipping or retention in a target pre-mRNA [9]. We also provide reference AOs optimised to modulate ubiquitously 
expressed human gene transcripts that may be employed as controls to monitor the efficiency of transfection, RNA extraction, and RT-PCR amplification.

Splice modulating AOs are designed to anneal to elements within or flanking an exon and influence its recognition by the spliceosome so that the exon is preferentially retained or excised from the mature mRNA as required. Redirection of splicing is presumably a consequence of preventing positive (enhancer) or negative (silencer) splicing factors from recognising enhancer or silencer elements in the pre-mRNA transcript. Steric hindrance at these sites alters the recognition of normal splice sites by the splicing machinery and leads to alternative selection of exons or intronic sequences in the targeted transcript [10]. Web-based tools such as SpliceAid 2 [11], Human Splicing Finder [12], and RegRNA [13] have facilitated the prediction of potential splice factor motifs in any given sequence. Bioinformatics can contribute to the design of splice switching AOs [14,15]. While it is relatively straightforward to target AOs to predicted enhancer or silencer motifs, or to confirmed splice donor or acceptor sites [14-16], this approach does not consistently yield effective splice altering AO sequences. Although the original AO that induced specific dystrophin exon 23 skipping in $m d x$ mouse muscle was directed to the donor splice site [17], AOs targeting the same coordinates of the human dystrophin transcript were completely ineffective [18]. Similarly, targeting the human dystrophin exon 51 donor splice site with AOs of different lengths and chemistries did not induce any exon skipping. Ultimately, identifying target domains within a pre-mRNA that influence splicing and then refining AO design through micro-walking must be done empirically.

To date, our laboratory has screened over 5,000 AOs directed at numerous gene transcripts, linked to genetic diseases that may be potentially amenable to a splice intervention therapy. In addition, we are also exploring non-productive splicing to downregulate expression of selected gene transcripts through inducing non-functional isoforms by either excising exons encoding crucial functional domains or disrupting the reading frame. Consequently, we have developed general guidelines that are efficient and effective in developing biologically active splice switching antisense oligomers.

1. The pre-mRNA sequence is interrogated by one or more in silico prediction programs to identify potential splice enhancer or silencer motifs.

2. Antisense oligonucleotides, typically 20 to 25 mers, are designed to anneal to the target motifs and synthesised as 2'-O-methyl (2-OMe) modified bases on a phosphorothioate (PS) backbone.

3. The test compounds are complexed with cationic liposome preparations and transfected into cells.

4. After incubation, total RNA is extracted and the target transcript is amplified using RT-PCR to assess differences in pre-mRNA processing, with and without $\mathrm{AO}$ treatment.

5. Oligomers shown to induce the desired changes in pre-mRNA processing are further refined by micro-walking around the annealing site and/or altering $\mathrm{AO}$ length.

6. Transfection studies over a range of concentrations are performed to identify compound(s) that modify splicing in a dose-dependent manner, and at the lowest concentration.

Oligomers composed of 2-OMe PS can be either sourced commercially from a variety of suppliers or, in our case, synthesised in-house on a nucleic acid synthesiser such as an Expedite 8909 or Akta OligoPilot plus 10. The 2-OMe PS AOs are excellent research tools as they are relatively easily transfected into many different cultured cells with the aid of cationic lipoplexes such as Lipofectamine ${ }^{\mathrm{TM}}$ 3000, Lipofectin ${ }^{\mathrm{TM}}$, and Lipofectamine ${ }^{\mathrm{TM}}$ 2000. However, when subsequent protein or functional studies are required, the optimised sequences are generally synthesised as phosphorodiamidate morpholino oligomers (PMOs), as this chemistry offers more efficient and sustained splicing modification and protein isoform expression without non-specific effects [19]. The PMO chemistry is well tolerated both in vitro and in vivo, even at relatively high dosages [20] and is our preferred oligomer chemistry for in vivo evaluation in animal studies and ultimately, for clinical application.

Unless directly coupled to a cell-penetrating agent, most AOs require a transfection reagent or protocol for efficient delivery into cultured cells, and in the case of splice switching compounds, be taken up into the nucleus where pre-mRNA processing occurs. Most commonly, a lipid-based solution is 
complexed with the AO prior to transfection [21], as the creation of a cationic lipid-complex capable of entering the cell via endocytosis vastly increases the AO uptake into the cell, compared to a gymnotic AO uptake. For screening of 2-OMe PS AOs, we typically use commercially available lipid transfection reagents, depending on the cell type. We have previously described transfection methods for efficient delivery of PMOs into cultured cells [22]. In addition to the transfection reagent or protocol, other factors that affect $\mathrm{AO}$ transfection efficiency in vitro include cell type and hence expression of the target gene, cell passage number and growth stage in culture, differentiation status, cell density, media and cell morphology during transfection, and $\mathrm{AO}$ chemistry and sequence composition. Here, we describe in vitro methods for $\mathrm{AO}$ design and the factors to consider during an initial screen of the most effective splice modifying antisense sequences using 2-OMe PS.

\section{Results}

Guidelines for Developing Splice Switching AOs

\section{Step 1-Selection of Target Motifs}

Once a target gene transcript and strategy is identified (e.g., exon skipping to remove a compromised exon from a disease causing gene, or disrupt expression of a target gene), open source web-based bioinformatics tools SpliceAid 2 [11] and Human Splicing Finder [12] may be used to identify predicted splice motifs, such as exon splice enhancers or exon splice silencers (Figure 1). Antisense oligomers complementary to the potential splice-associated motifs will then be synthesised as 2-OMe PS AOs. To induce exon skipping, the canonical acceptor and donor splice sites are obvious and well-defined targets, and since most exons are less than 200 bases in length, an additional three or four AOs, typically 20-25 nucleotides in length, provide reasonable coverage in the first pass.

(A)

\section{Human Splicing Finder 3.1} ATXN3 - Exon 9
(B)

Splice Aid 2.0

ATXN3 - Exon 9

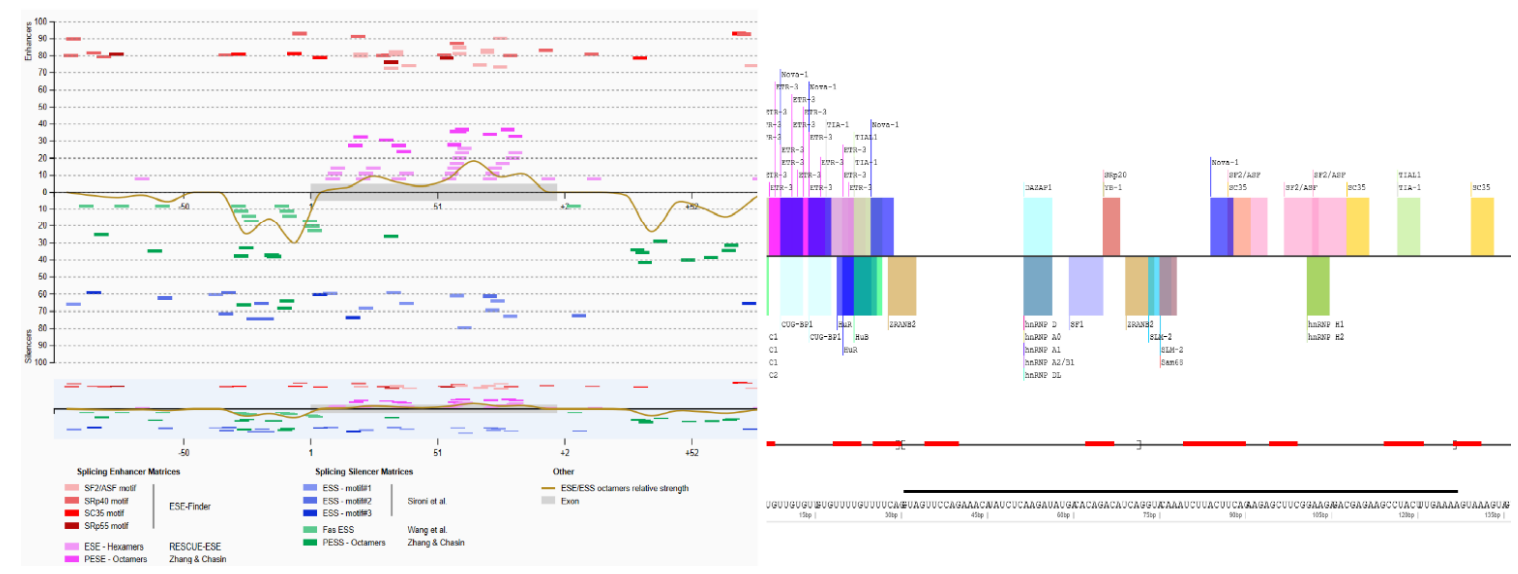

Figure 1. In silico prediction of splice motifs for exon 9 of the ATXN3 transcript using Human Splicing Finder (A) and SpliceAid 2 (B). Exon position is identified by the black line above the sequence in SpliceAid 2.

We have reported a nomenclature system for the AOs according to gene, species, exon number, and annealing coordinates [23] (Figure 2). The nomenclature begins with the name of the transcript (e.g., survival of motor neuron 1; SMN1), then the species of the target mRNA (e.g., H: human or M: mouse), followed by the target exon number of the specified transcript and specification of an acceptor (A) or donor (D) site. The annealing coordinates are shown in brackets from the $5^{\prime}$ to $3^{\prime}$ position within the pre-mRNA transcript. The intronic bases are designated with a negative prefix (-) and the exonic position with a positive $(+)$ symbol. The annealing coordinates are the positions of bases relative to the 
acceptor or donor sites of the reference transcript as denoted by National Center for Biotechnology Information and Ensembl genome browser 96. It is important to describe the reference transcript ID, especially in the case of targeting gene transcript isoforms that are composed of different numbers of exons. This $\mathrm{AO}$ nomenclature provides researchers with a unique designation, conveying the precise annealing coordinates of the targeted transcript that is particularly relevant when refining AO design by micro-walking around a responsive annealing site. Subtle shifts in $\mathrm{AO}$ annealing coordinates or length are immediately apparent and facilitates optimal AO design.

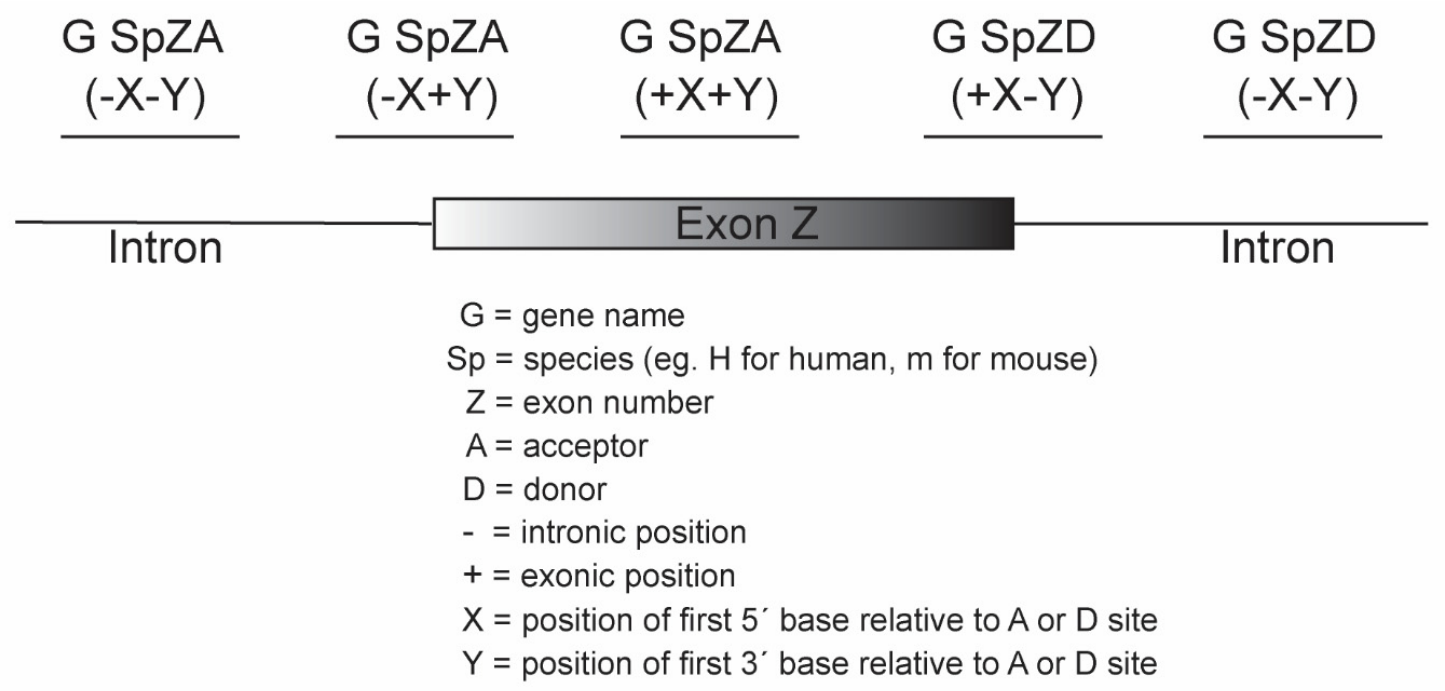

Figure 2. Nomenclature for antisense oligonucleotides.

Step 2-Choice of Cell Type

When possible, screening and evaluation of splice switching AOs should be performed in cells expressing the target gene transcript and protein. GeneCards [24] offers a quick and convenient guide to cell and tissue-specific gene expression. However, certain disease-affected cell types-e.g., motor neurons or photoreceptors-may be difficult, impractical, or impossible to obtain and propagate from living patients. Consequently, it may be necessary to initiate $\mathrm{AO}$ design and evaluation in patient-derived lymphocytes or skin fibroblasts, and while not optimal, these cell types are relatively easy to obtain and culture. Although target gene expression may be relatively low in lymphocytes or fibroblasts, it may be sufficient for initial proof of concept studies at the RNA level.

When studying most muscle diseases, patient-derived myoblasts would be the preferred material for study. However, obtaining muscle biopsies for the propagation of myogenic cells requires more invasive procedures compared to collecting a skin punch or blood sample. Unless elective surgery has been scheduled, working with healthy or patient-derived myogenic cells should only be considered after careful deliberation and consultation. Dermal fibroblasts can be propagated and subsequently induced into the myogenic lineage using MyoD expressing vectors, a common method routinely used to differentiate fibroblasts into myogenic cells [25-27].

In some instances, it is not necessary to use patient cells for the design of potentially therapeutic AOs. For example, the most common type of Duchenne muscular dystrophy-causing mutation is the genomic deletion of one or more exons, with subsequent disruption of the open reading frame. Since a normal exon flanking the frame-shifting deletion must be excised to restore the reading frame, the current suite of dystrophin exon skipping oligomers were designed against the normal dystrophin sequence and first evaluated in cells derived from healthy individuals $[1,2,8]$. Furthermore, AO optimisation in healthy cells sets a high standard, since the full complement of splicing factors are present in the context of the normal transcript, and the transcript induced by skipping a frame-shifting exon will be out of frame and hence subject to nonsense-mediated decay. While developing AOs to 
excise human dystrophin exon 8, compounds were first evaluated using healthy myoblasts, and clear differences in exon skipping efficiencies were readily evident. However, when these same compounds were tested in amenable patient-derived myogenic cells (e.g., missing exons 3-7 or 5-7), the distinction between poor and robust exon skipping AOs was much less evident [28]. For AOs designed to downregulate expression of a target protein by disrupting normal splicing, screening can again be undertaken initially in healthy cell lines. Once optimised, proof of concept studies can then be initiated in patient-derived cells for further validation and protein studies.

For gene transcripts not expressed in either fibroblasts, lymphocytes or myoblasts, other commercially available cell lines, such as HEK293 (human embryonic kidney epithelium) or SH-SY5Y (neuroblastoma) lines available from repositories (Coriell Institute for Medical Research or American Type Culture Collection), may suffice. However, for specific disease-causing mutations, patient-derived cells are required for testing and validation of the $\mathrm{AO}$ and assessing the mutation specific effect. The construction of mini-gene assays to study the consequences of a particular splice mutation and $\mathrm{AO}$ intervention can be helpful. However, the utility of mini-gene assays can be limited by the length and structure of the cloned exonic and intronic sequences, and the cell type or strain used.

\section{Step 3-Delivery Reagents}

Once the AOs are designed and an appropriate cell type is chosen, we recommend exploring different transfection reagents for optimal AO delivery and uptake. Depending on the mechanism of action, AOs are required to be delivered to either the cytoplasm (for protein translation blockade and RNase $\mathrm{H}$ mediated mRNA degradation) or the nucleus (to alter pre-mRNA processing, including splicing or polyadenylation). Oligomers labelled with various fluorophores (e.g., FAM and TET) may be used to assess gross transfection efficiency, distribution, and uptake of each reagent; however, when exploring novel splice modification approaches, we recommend using a validated splice modulating AO as a control. As shown in Figure 3, one such control is an AO designed to induce skipping of exon 3 from the ITGA4 transcript, a widely expressed gene in many different cell types. Not only will transfection of a control AO provide a guide to the transfection efficiencies, it can also be useful in assessing RNA quality and quantity in conjunction with the RT-PCR assays.

The ITGA4 transcript in healthy human fibroblasts was analysed by end point RT-PCR. The cells were transfected with 100 nM ITGA4 H3A (+ $30+49$ ), a 2-OMe PS AO that induces skipping of exons 3 and 4 from the ITGA4 gene transcript, using three different lipid-based transfection reagents (Figure $3 \mathrm{~A}$ ). Although all transfection reagents tested did deliver the $\mathrm{AO}$, the transfection using Lipofectamine ${ }^{\mathrm{TM}}$ 3000 reagent induced the highest level of exon skipping in these cells. Lipofectamine ${ }^{\mathrm{TM}}$ RNAiMax showed a similar exon skipping pattern as Lipofectamine ${ }^{\mathrm{TM}} 3000$, however, higher levels of cell death were induced by the former. The recommended lipid-based transfection reagents for delivery of 2-OMe PS AOs into the cell lines tested in our laboratory are listed in Table 1. Once the optimal transfection reagent is identified for a particular cell type, evaluation of $\mathrm{AO}$ sequences can proceed.

Table 1. Recommended transfection reagents for different cell lines

\begin{tabular}{|c|c|}
\hline Cell Lines & Transfection Reagents \\
\hline Dermal fibroblasts & Lipofectin $^{\mathrm{TM}}$, Lipofectamine ${ }^{\mathrm{TM}} 3000$ \\
\hline Myoblasts and myotubes & Lipofectamine $^{\mathrm{TM}} 2000$ \\
\hline Lymphoblasts and lymphocytes & Nucleofection P3 Primary Cell Kit \\
\hline Huh7 & Lipofectamine $^{\mathrm{TM}}$ 3000, Lipofectamine ${ }^{\mathrm{TM}}$ RNAiMax \\
\hline HEK293 & Lipofectamine $^{\mathrm{TM}} 3000$ \\
\hline $\mathrm{H} 2 \mathrm{k} m d x$ & Lipofectin $^{\mathrm{TM}}$ \\
\hline MO3.13 & Lipofectamine $^{\mathrm{TM}} 3000$ \\
\hline iPSCs and neural stem cells & Lipofectamine $^{\mathrm{TM}}$ Stem \\
\hline
\end{tabular}

Note: When evaluating AOs for splice modulation in primary cells, it is preferable to use cultures of lower passage number as we found that cultures with higher passage numbers tend to be transfected with lower efficiencies. 
One important parameter to consider when designing primers for RT-PCR analysis of the full-length and AO-induced transcripts is to place the forward and reverse primers a few exons away from the targeted exon. We have now encountered several examples where targeting one exon for exclusion from the mature mRNA also influences recognition and retention of flanking exons and introns. As shown in Figure 3, amplification of the ITGA4 transcript from exons 1 to 10 (Figure 3A) showed robust exon skipping, but not when amplified from exon 1 to 4 after transfection with the same AO designed to skip exon 3 (Figure 3B).

(A) Amplicon exon 1 to 10

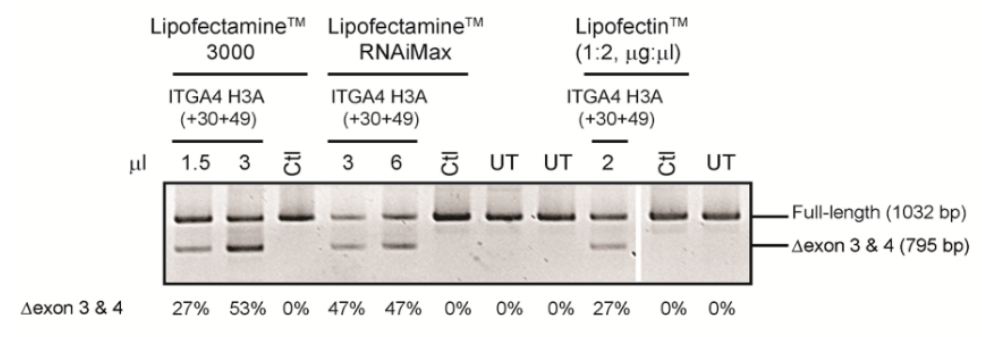

(B) Amplicon exon 1 to 4

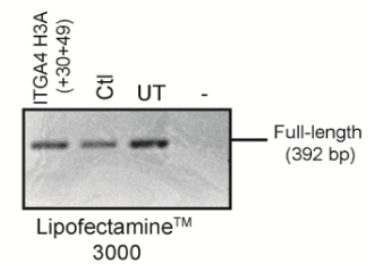

Figure 3. Modification of integrin alpha 4 (ITGA4) transcripts after healthy human fibroblasts were transfected with different lipid-based transfection reagents. (A) Three lipid-based reagents were used to transfect cells with ITGA4 H3A $(+30+39)$ at $100 \mathrm{nM}$ for $24 \mathrm{hr}$. Total RNA was extracted and RT-PCR was undertaken across exons 1 to 10 of the ITGA4 transcripts. Transfection reagent volumes are indicated above the gel. Ctl; control AO that does not anneal to any sequence, UT; untreated, -; no template control. Percentages of ITGA4 exon 3 and 4 skipping are indicated below the gel. The control AO was transfected using the maximum volume of transfection reagents. (B) RT-PCR amplification of the ITGA4 transcript across exons 1 to 4 from RNA extracted from sample transfected with Lipofectamine 3000 from (A).

Step 4-Initial AO Screen

In early AO splice switching studies, the use of negative AO control sequences-either random, scrambled, or unrelated sequences-was essential to confirm specific target modification. Establishing target specificity is particularly crucial in situations where gene downregulation is the desired outcome. However, in many cases of splice switching, either exon skipping, exon retention or intron retention, the presence of a novel transcript is proof of the anticipated antisense mechanism. When AOs designed to a specific target do not affect the processing of that specific gene transcript, it is likely (but not inconceivable) that imperfect annealing to another pre-mRNA would have a minimal, if any effect.

Depending upon the gene and targeted exon, it has been our experience that up to two out of three AOs designed in a first pass can induce some level of exon skipping. However, targeting certain motifs noticeably results in more efficient exon skipping than others, and when developing any AO for clinical use, it is obvious that the most appropriate compound will be one that induces robust splice switching at a low concentration. The use of a positive transfection control AO is recommended for each transfection experiment, as this can control for transfection efficiencies across different experiments. It is also important to note that cell confluency, passage number, and other culture conditions can substantially influence transfection efficiency in primary cells and may lead to variations in AO efficacy between biological replicates.

In some cases, individual AOs are ineffective at modifying exon selection, even after transfection at high concentrations. We have frequently found that selective $\mathrm{AO}$ cocktails, which include two or more AOs used in conjunction for a given exon target, mediate exon skipping in a synergistic manner, while each AO transfected alone is ineffective [18,29]. Conversely, we have also observed a marked decrease in exon skipping efficiency when two highly effective AOs are combined.

Note: It is recommended to confirm the identity of novel 'exon skipped' products by direct DNA sequencing, as nearby cryptic splice sites may be activated and generate amplicons of a similar 
length to the expected product. A difference of only a few bases in length can be difficult to resolve on an agarose gel, and such differences would be impossible to detect in longer RT-PCR products representing multiple exons [30].

Upon identification of amenable sites in the pre-mRNA that induce the desired splice modulation, AOs can be further optimised by 'micro-walking' and shifting the $\mathrm{AO}$ annealing sites in either direction to ensure the most amenable splice motifs have been targeted. An example of micro-walking is illustrated in Figure 4. Generally, to find the most effective AO, the annealing sites are moved five nucleotides in either the $5^{\prime}$ or $3^{\prime}$ direction, while retaining the same AO length. As shown in Figure 4, secondary screening of the AOs, ITGA4 H3A $(+41+65)$ and ITGA4 H3A (+ 51 + 75), marginally improved exon skipping efficiency, compared to ITGA4 H3A $(+46+70)$ indicating this general region would be suitable as an AO target. Alternatively, shifting the annealing site targeted by ITGA4 H3D $(+6-19)$ further into the intron with ITGA4 H3D (+ 1 - 24) improved exon skipping efficiency from $18 \%$ to $34 \%$. If considered necessary and of particular relevance, further micro-walking could be undertaken via moving the lead AO candidate target sequence by a few nucleotides in either the $5^{\prime}$ or $3^{\prime}$ direction. As a final optimisation step, once the most responsive or amenable annealing site is defined, the AO length may be truncated from either end. Shorter AOs are not only more efficiently synthesized, but substantially less costly to produce, an important consideration that will influence eventual clinical implementation. In some cases, AOs longer than 25 bases may be justified and must be considered on a case-by-case basis. We showed that efficient dystrophin exon 16 could be induced by overlapping 25 mers but increasing the length to a 30 mer resulted in a four-fold increase in exon skipping efficiency [31]. Hence, a 20\% increase in AO length (and cost) resulted in a $400 \%$ increase in potency as assessed in vitro, thus allowing cost: benefits to be assessed.

(A)

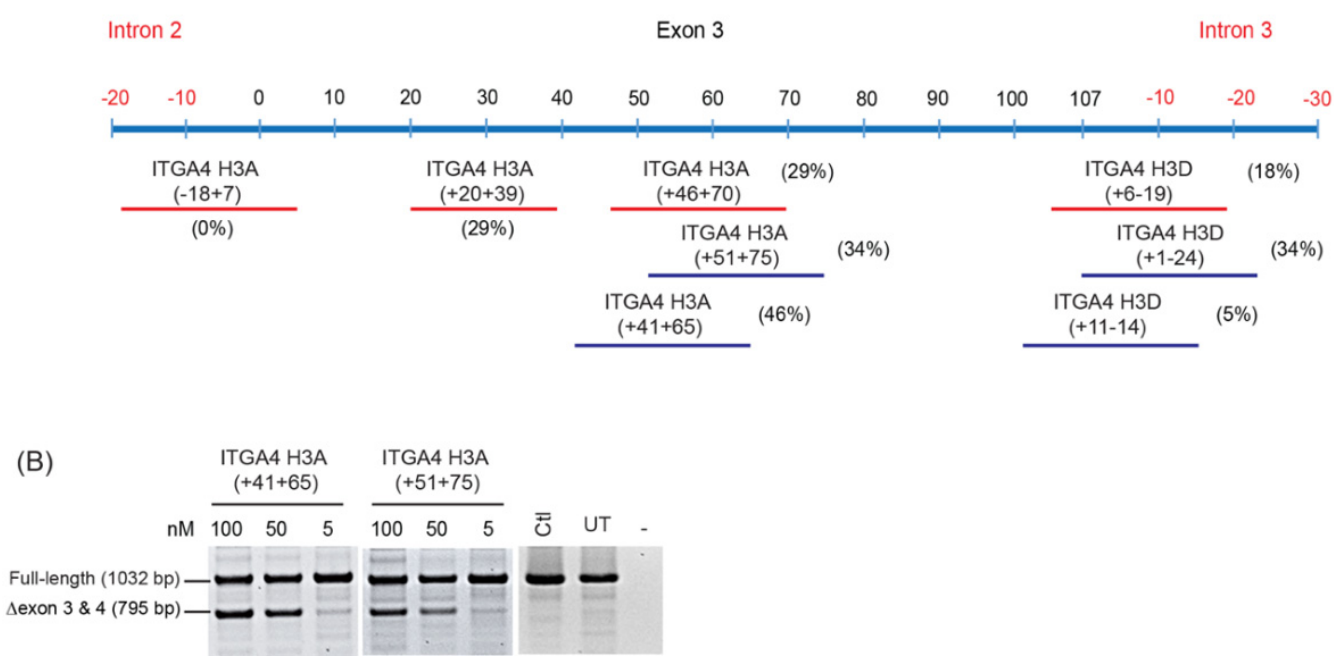

Figure 4. (A) RT-PCR analysis of ITGA4 transcripts demonstrating refinement of splice switching AOs targeting ITGA4 exon 3. Oligomers tested in the first screen are indicated by red lines and the micro-walked AOs tested in the second screen are represented by blue lines. Levels of exon skipping after transfection at $100 \mathrm{nM}$ are indicated in brackets. (B) Comparison of two AOs that induce skipping of ITGA4 exon 3 and 4 at various concentrations (100, 50, and $5 \mathrm{nM})$. Ctl; control AO that does not anneal to any sequence, UT; untreated, -; no template control.

As a final evaluation to demonstrate reproducibility and efficacy, AO titrations should be performed to discriminate between AOs that induce similar levels of exon skipping, as shown in Figure 4B. Both ITGA4 H3A (+ $41+65)$ and ITGA4 H3A (+ 51 + 75) resulted in efficient exon skipping at $100 \mathrm{nM}$. However, when both AOs were transfected at $50 \mathrm{nM}$, the lower efficiency of ITGA4 H3A (+ 51 + 75) compared to ITGA4 H3A $(+41+65)$ was evident. 


\section{Discussion}

Here we describe general guidelines for developing, screening, and refining splice modulating $\mathrm{AO}$ sequences. It has been our experience that within a given gene transcript, some exons are readily excised from the mature mRNA, albeit at variable efficiencies in vitro, whereas other exons in the same transcript are more resistant to exon skipping [22,29]. In some cases, there may be either only a single region found to mediate AO-induced exon skipping, or combinations of AOs are needed to induce exon skipping, with the combinations sometimes acting synergistically [29]. We are yet to determine parameters that predict the ideal exon or transcript target for the design of splice modulating AOs, and experimental optimisation is therefore critical to the development of AO therapeutics.

As with all forms of genetic therapies, AO delivery is a crucial aspect for splice switching efficacy. Relatively minor changes to the transfection protocol can dramatically improve or weaken apparent AO splice switching efficiency, and consequently, we suggest that before any AO is classified as ineffective in modulating splicing, various transfection methods should be evaluated. We describe an AO sequence that can be used as a positive control to modify the expression of the ITGA4 transcript, widely expressed in most cell types. We suggest the use of this AO as a positive transfection control, an important inclusion during the initial stages of AO strategy design. A positive control AO allows one to monitor the experimental protocols (e.g., transfection, RNA extraction, RT-PCR).

Oligomer synthesis demands high coupling efficiencies and hence oligomer length can be an important and significant consideration in AO drug design, highlighting the need to keep the AO length as short as possible while still maintaining adequate exon skipping levels. Some sequences can be challenging to synthesise, for example AOs with a high GC content $(>75 \%)$ and sequences that include stretches of four Gs have the ability to form G-quartets [32]; structures that stack on top of each other and form tetrad-helical structures, thus severely inhibiting the functionality and solubility of the oligomer [33].

One crucial aspect of developing splice modulating AOs is to enhance stability in biological systems without compromising efficiency and introducing toxicity. At present, 2-OMe PS AOs are cost-effective for initial screening. However, in several studies, the oligonucleotides on a PS backbone have shown toxicity, off-target effects, and injection site reactions [34-37]. Nevertheless, these negatively charged AOs are ideal as research tools as they can be readily transfected into cultured cells as cationic lipoplexes. The lead AO sequences identified by 2-OMe PS AO screens may prove more effective when synthesised as the clinically safe PMO chemistry. There are no reports of serious adverse events occurring after long-term treatment with Exondys 51 [1,2], while the same cannot be said for the 2-OMe PS AO drug Drisapersen. PMOs escape the electrostatic repulsion from the negatively charged RNA or DNA due to their neutral backbone $[20,38]$. This enables much higher binding specificity and affinity when compared to 2-OMe PS AOs. Furthermore, PMOs exert little to no off-target effects or non-specific binding, which is largely attributed to their neutral charge [20].

In conclusion, we have described a robust approach for developing and designing splice modulating AOs that may eventually enter the clinic. Extensive refining of the AO sequences to achieve the shortest oligomer with high efficacy is crucial to reduce the cost and production issues of the candidate oligo sequences. Antisense oligomer-mediated modulation of gene transcripts involved in genetic diseases has great potential for therapeutic application. The systematic evaluation of AOs in the manner we describe here will ensure the selection of the most efficacious and safe AOs for clinical trials.

\section{Materials and Methods}

\subsection{Cell Culture}

All cell culture reagents were purchased from Gibco, ThermoFisher Scientific, (Scoresby, Victoria, Australia) unless otherwise stated. Primary dermal fibroblasts obtained from a healthy volunteer, with informed consent (approved by the Murdoch University Human Research Ethics Committee, 
approval number 2013/156, 25 October 2013) were propagated in Dulbecco's modified Eagle medium (DMEM) supplemented with L-Glutamine and 10\% foetal bovine serum (FBS) (Scientifix, Cheltenham, Victoria, Australia).

\subsection{Antisense Oligonucleotides (AOs)}

Antisense oligonucleotides comprising 2'-O-methyl modified bases on a phosphorothioate backbone (2-OMe PS) were synthesised in-house on an Expedite 8909 Nucleic Acid synthesiser (Applied Biosystems, Melbourne, Victoria, Australia) using the $1 \mu \mathrm{mol}$ thioate synthesis protocol as described previously [39]. Briefly, phenyl acetyl disulphide was used in the sulphrisation of the oligonucleotide. The 2'-hydroxyl positions are protected with t-butyldimethylsilyl group. Specifically, benzoyl was used as the protection reagent for nucleotide A and $C$ monomers, while isobutyryl was used for nucleotide $G$ monomer and $U$ monomer not requiring protection. After synthesis, the oligonucleotides were cleaved from the support following incubation in ammonium hydroxide for a minimum of $16 \mathrm{~h}$ at room temperature. The AOs were subsequently desalted under sterile conditions using the NAP-10 columns (GE Healthcare, Sydney, NSW, Australia) according to manufacturer's instructions. A list of 2-OMe PS AOs used in this study are summarised in Table 2.

\subsection{2'-O-Methyl Phosphorothioate AO Transfection}

Approximately 15,000 fibroblasts were seeded onto 24-well tissue culture plates and incubated overnight at $37^{\circ} \mathrm{C}$ in fibroblast propagation media. When approximately $70-80 \%$ confluent, cells were transfected with 2-OMe PS AOs using Lipofectamine ${ }^{\mathrm{TM}} 3000$, Lipofectamine ${ }^{\mathrm{TM}}$ RNAiMax, or Lipofectin ${ }^{\mathrm{TM}}$ transfection reagent according to the manufacturer protocols. Opti-MEM media was used for all transfections. Transfected cells were incubated at $37^{\circ} \mathrm{C}$ for $24 \mathrm{~h}$ before RNA was extracted for transcript analysis.

\subsection{RT-PCR}

Total RNA was extracted using Trizol (Life Technologies, Scoresby, Victoria, Australia) according to manufacturer's guidelines for transcript analysis. RT-PCR was performed using RNA (50 ng) from AO-treated and untreated cells and a Superscript III One-Step RT-PCR System (Life Technologies, Australia). Primer sequences for all RT-PCR primers used in this study can be found in Table 3. RT-PCR amplification was performed using the following thermocycling conditions: $55^{\circ} \mathrm{C}$ for $30 \mathrm{~min}, 30$ cycles of $94{ }^{\circ} \mathrm{C}$ for $30 \mathrm{~s}, 55^{\circ} \mathrm{C}$ for $30 \mathrm{~s}$, and $68^{\circ} \mathrm{C}$ for $2 \mathrm{~min}$. RT-PCR products were resolved on $2 \%$ agarose gels in Tris-acetate EDTA buffer and images were captured using a Fusion-FX gel documentation system (Vilber Lourmat, Eberhardzell, France). Product identity was confirmed by reamplification and purification of separated amplicons [40], followed by Sanger sequencing by the Australian Genome Research Facility (AGRF).

Table 2. List of AOs [41].

\begin{tabular}{cc}
\hline Name & Sequence $\mathbf{( 5}^{\prime}-\mathbf{3}^{\prime}$ ) \\
\hline ITGA4 H3A $(+30+49)$ & UCUCUCUCUUCCAAACAAGU \\
\hline ITGA4 H3A $(-18+7)$ & GGGCUACCUAUAGCAUGUGAAAAUA \\
\hline ITGA4 H3A $(+20+39)$ & CCAAACAAGUCUUUCCACAA \\
\hline ITGA4 H3A $(+46+70)$ & GUGACCCCCAACCACUGAUUGUCUC \\
\hline ITGA4 H3A $(+41+65)$ & CCCCAACCACUGAUUGUCUCUCUCU \\
\hline ITGA4 H3A $(+51+75)$ & AAAGUGUGACCCCCAACCACUGAUU \\
\hline ITGA4 H3D $(+6-19)$ & GACCAGUUCCAAUACCUACCACGAU \\
\hline ITGA4 H3D $(+11-14)$ & GUUCCAAUACCUACCACGAUGGAUC \\
\hline ITGA4 H3D $(+1-24)$ & CUGUGGACCAGUUCCAAUACCUACC \\
\hline Ctl & GGAUGUCCUGAGUCUAGACCCUCCG \\
\hline
\end{tabular}


Table 3. List of primers used in this study.

\begin{tabular}{|c|c|c|}
\hline Name & Sequence $\left(5^{\prime}-3^{\prime}\right)$ & $\begin{array}{c}\text { Amplification Performed after Treatment with the } \\
\text { Following AOs }\end{array}$ \\
\hline $\begin{array}{l}\text { ITGA4 ex1_F } \\
\text { ITGA4 ex10_R }\end{array}$ & $\begin{array}{l}\text { gagagcgcgctgctttaccagg } \\
\text { gccatcattgtcaatgtcgcca }\end{array}$ & All AOs \\
\hline $\begin{array}{l}\text { ITGA4 ex1_F } \\
\text { ITGA4 ex4_R }\end{array}$ & $\begin{array}{l}\text { gagagcgcgctgctttaccagg } \\
\text { ggcactccatagcaaccacc }\end{array}$ & ITGA4 H3A(+30+49) \\
\hline
\end{tabular}

Author Contributions: Conceptualisation, M.T.A.-H. and C.S.M.; Methodology, M.T.A.-H., C.S.M., K.A.H., and S.D.W.; Formal Analysis: M.T.A.-H., C.S.M., and K.A.H.; Investigation, M.T.A.-H., C.S.M., and K.A.H.; Writing, M.T.A.-H., C.S.M., K.A.H., S.F., and S.D.W.; Editing, M.T.A.-H., C.S.M., K.A.H., I.L.P., L.L.F., K.G., S.F., and S.D.W.; Supervision, S.F. and S.D.W.; Resources, S.F. and S.D.W.; Funding Acquisition, S.F. and S.D.W.

Funding: Funding received from the NHMRC (AP1144791).

Conflicts of Interest: S.F. and S.D.W. are consultants for Sarepta Therapeutics, and M.T.A.-H.'s salary is partly supported by Sarepta Therapeutics. This association has not influenced this work in any fashion.

\section{References}

1. Mendell, J.R.; Goemans, N.; Lowes, L.P.; Alfano, L.N.; Berry, K.; Shao, J.; Kaye, E.M.; Mercuri, E.; Eteplirsen Study Group; Telethon Foundation DMD Italian Networkc; et al. Longitudinal effect of eteplirsen versus historical control on ambulation in D uchenne muscular dystrophy. Ann. Neurol. 2016, 79, $257-271$. [CrossRef] [PubMed]

2. Mendell, J.R.; Sahenk, Z.; Rodino-Klapac, L.R. Clinical trials of exon skipping in duchenne muscular dystrophy. Expert Opin. Orphan Drugs 2017, 5, 683-690. [CrossRef]

3. Finkel, R.S.; Chiriboga, C.A.; Vajsar, J.; Day, J.W.; Montes, J.; De Vivo, D.C.; Yamashita, M.; Rigo, F.; Hung, G.; Schneider, E. Treatment of infantile-onset spinal muscular atrophy with nusinersen: A phase 2, open-label, dose-escalation study. Lancet 2017, 388, 3017-3026. [CrossRef]

4. Rinaldi, C.; Wood, M.J. Antisense oligonucleotides: The next frontier for treatment of neurological disorders. Nat. Rev. Neurol. 2018, 14, 9-21. [CrossRef] [PubMed]

5. Scoles, D.R.; Meera, P.; Schneider, M.D.; Paul, S.; Dansithong, W.; Figueroa, K.P.; Hung, G.; Rigo, F.; Bennett, C.F.; Otis, T.S. Antisense oligonucleotide therapy for spinocerebellar ataxia type 2. Nature 2017, 544, 362-366. [CrossRef] [PubMed]

6. Stein, C.; Hansen, J.B.; Lai, J.; Wu, S.; Voskresenskiy, A.; Høg, A.; Worm, J.; Hedtjärn, M.; Souleimanian, N.; Miller, P. Efficient gene silencing by delivery of locked nucleic acid antisense oligonucleotides, unassisted by transfection reagents. Nucleic Acids Res. 2009, 38, e3. [CrossRef] [PubMed]

7. Julien, T.; Frankel, B.; Longo, S.; Kyle, M.; Gibson, S.; Shillitoe, E.; Ryken, T. Antisense-mediated inhibition of the bcl-2 gene induces apoptosis in human malignant glioma. Surg. Neurol. 2000, 53, 360-369. [CrossRef]

8. Mendell, J.R.; Rodino-Klapac, L.R.; Sahenk, Z.; Roush, K.; Bird, L.; Lowes, L.P.; Alfano, L.; Gomez, A.M.; Lewis, S.; Kota, J. Eteplirsen for the treatment of duchenne muscular dystrophy. Ann. Neurol. 2013, 74, 637-647. [CrossRef]

9. Havens, M.A.; Hastings, M.L. Splice-switching antisense oligonucleotides as therapeutic drugs. Nucleic Acids Res. 2016, 44, 6549-6563. [CrossRef]

10. Flynn, L.L.; Mitrpant, C.; Pitout, I.L.; Fletcher, S.; Wilton, S.D. Antisense oligonucleotide-mediated terminal intron retention of the smn2 transcript. Mol. Ther. Nucleic Acids 2018, 11, 91-102. [CrossRef]

11. Piva, F.; Giulietti, M.; Burini, A.B.; Principato, G. Spliceaid 2: A database of human splicing factors expression data and rna target motifs. Hum. Mutat. 2012, 33, 81-85. [CrossRef] [PubMed]

12. Desmet, F.-O.; Hamroun, D.; Lalande, M.; Collod-Béroud, G.; Claustres, M.; Béroud, C. Human splicing finder: An online bioinformatics tool to predict splicing signals. Nucleic Acids Res. 2009, 37, e67. [CrossRef] [PubMed]

13. Huang, H.-Y.; Chien, C.-H.; Jen, K.-H.; Huang, H.-D. Regrna: An integrated web server for identifying regulatory rna motifs and elements. Nucleic Acids Res. 2006, 34, W429-W434. [CrossRef] [PubMed]

14. Aartsma-Rus, A. Overview on aon design. In Exon Skipping; Springer: Heidelberg, Germany, 2012; pp. 117-129. 
15. Pramono, Z.A.D.; Wee, K.B.; Wang, J.L.; Chen, Y.J.; Xiong, Q.B.; Lai, P.S.; Yee, W.C. A prospective study in the rational design of efficient antisense oligonucleotides for exon skipping in the dmd gene. Hum. Gene Ther. 2012, 23, 781-790. [CrossRef] [PubMed]

16. Aartsma-Rus, A.; Van Vliet, L.; Hirschi, M.; Janson, A.A.; Heemskerk, H.; De Winter, C.L.; De Kimpe, S.; Van Deutekom, J.C.; Ac't Hoen, P.; van Ommen, G.-J.B. Guidelines for antisense oligonucleotide design and insight into splice-modulating mechanisms. Mol. Ther. 2009, 17, 548-553. [CrossRef] [PubMed]

17. Gebski, B.L.; Mann, C.J.; Fletcher, S.; Wilton, S.D. Morpholino antisense oligonucleotide induced dystrophin exon 23 skipping in mdx mouse muscle. Hum. Mol. Genet. 2003, 12, 1801-1811. [CrossRef]

18. Mitrpant, C.; Adams, A.M.; Meloni, P.L.; Muntoni, F.; Fletcher, S.; Wilton, S.D. Rational design of antisense oligomers to induce dystrophin exon skipping. Mol. Ther. 2009, 17, 1418-1426. [CrossRef]

19. Greer, K.L.; Lochmüller, H.; Flanigan, K.; Fletcher, S.; Wilton, S.D. Targeted exon skipping to correct exon duplications in the dystrophin gene. Mol. Ther. Nucleic Acids 2014, 3, e155. [CrossRef]

20. Summerton, J.E. Morpholino, sirna, and s-DNA compared: Impact of structure and mechanism of action on off-target effects and sequence specificity. Curr. Top. Med. Chem. 2007, 7, 651-660. [CrossRef]

21. Zuhorn, I.S.; Engberts, J.B.; Hoekstra, D. Gene delivery by cationic lipid vectors: Overcoming cellular barriers. Eur. Biophys. J. 2007, 36, 349-362. [CrossRef]

22. Aung-Htut, M.T.; McIntosh, C.S.; West, K.A.; Fletcher, S.; Wilton, S.D. In vitro validation of phosphorodiamidate morpholino oligomers. Molecules 2019, 24, 2922. [CrossRef] [PubMed]

23. Mann, C.J.; Honeyman, K.; McClorey, G.; Fletcher, S.; Wilton, S.D. Improved antisense oligonucleotide induced exon skipping in the mdx mouse model of muscular dystrophy. J. Gene Med. Cross-Discip. J. Res. Sci. Gene Transf. Clin. Appl. 2002, 4, 644-654.

24. Weizmann-Insitite. Gene Cards Human Gene Database. Available online: https://www.genecards.org/ (accessed on 14 March 2019).

25. Murry, C.E.; Kay, M.A.; Bartosek, T.; Hauschka, S.D.; Schwartz, S.M. Muscle differentiation during repair of myocardial necrosis in rats via gene transfer with myod. J. Clin. Investig. 1996, 98, 2209-2217. [CrossRef] [PubMed]

26. Lattanzi, L.; Salvatori, G.; Coletta, M.; Sonnino, C.; De Angelis, M.C.; Gioglio, L.; Murry, C.E.; Kelly, R.; Ferrari, G.; Molinaro, M. High efficiency myogenic conversion of human fibroblasts by adenoviral vector-mediated myod gene transfer. An alternative strategy for ex vivo gene therapy of primary myopathies. J. Clin. Investig. 1998, 101, 2119-2128. [CrossRef] [PubMed]

27. Choi, J.; Costa, M.; Mermelstein, C.; Chagas, C.; Holtzer, S.; Holtzer, H. Myod converts primary dermal fibroblasts, chondroblasts, smooth muscle, and retinal pigmented epithelial cells into striated mononucleated myoblasts and multinucleated myotubes. Proc. Natl. Acad. Sci. USA 1990, 87, 7988-7992. [CrossRef] [PubMed]

28. Fletcher, S.; Adkin, C.F.; Meloni, P.; Wong, B.; Muntoni, F.; Kole, R.; Fragall, C.; Greer, K.; Johnsen, R.; Wilton, S.D. Targeted exon skipping to address "leaky" mutations in the dystrophin gene. Mol. Ther. Nucleic Acids 2012, 1, e48. [CrossRef] [PubMed]

29. Adams, A.M.; Harding, P.L.; Iversen, P.L.; Coleman, C.; Fletcher, S.; Wilton, S.D. Antisense oligonucleotide induced exon skipping and the dystrophin gene transcript: Cocktails and chemistries. BMC Mol. Biol. 2007, 8, 57. [CrossRef] [PubMed]

30. Fletcher, S.; Ly, T.; Duff, R.; Howell, J.M.; Wilton, S. Cryptic splicing involving the splice site mutation in the canine model of duchenne muscular dystrophy. Neuromuscul. Disord. 2001, 11, 239-243. [CrossRef]

31. Harding, P.; Fall, A.; Honeyman, K.; Fletcher, S.; Wilton, S. The influence of antisense oligonucleotide length on dystrophin exon skipping. Mol. Ther. 2007, 15, 157-166. [CrossRef]

32. Jing, N.; Li, Y.; Xiong, W.; Sha, W.; Jing, L.; Tweardy, D.J. G-quartet oligonucleotides: A new class of signal transducer and activator of transcription 3 inhibitors that suppresses growth of prostate and breast tumors through induction of apoptosis. Cancer Res. 2004, 64, 6603-6609. [CrossRef]

33. Williamson, J.R. G-quartet structures in telomeric DNA. Annu. Rev. Biophys. Biomol. Struct. 1994, $23,703-730$. [CrossRef] [PubMed]

34. Flanigan, K.M.; Voit, T.; Rosales, X.Q.; Servais, L.; Kraus, J.E.; Wardell, C.; Morgan, A.; Dorricott, S.; Nakielny, J.; Quarcoo, N. Pharmacokinetics and safety of single doses of drisapersen in non-ambulant subjects with duchenne muscular dystrophy: Results of a double-blind randomized clinical trial. Neuromuscul. Disord. 2014, 24, 16-24. [CrossRef] [PubMed] 
35. Dias, N.; Stein, C. Antisense oligonucleotides: Basic concepts and mechanisms. Mol. Cancer Ther. 2002, 1, 347-355. [PubMed]

36. Wong, E.; Goldberg, T. Mipomersen (kynamro): A novel antisense oligonucleotide inhibitor for the management of homozygous familial hypercholesterolemia. Pharm. Ther. 2014, 39, 119-122.

37. Chi, X.; Gatti, P.; Papoian, T. Safety of antisense oligonucleotide and sirna-based therapeutics. Drug Discov. Today 2017, 22, 823-833. [CrossRef]

38. Summerton, J.; Weller, D. Morpholino antisense oligomers: Design, preparation, and properties. Antisense Nucleic Acid Drug Dev. 1997, 7, 187-195. [CrossRef]

39. Wilton, S.D.; Fall, A.M.; Harding, P.L.; McClorey, G.; Coleman, C.; Fletcher, S. Antisense oligonucleotide-induced exon skipping across the human dystrophin gene transcript. Mol. Ther. 2007, 15, 1288-1296. [CrossRef]

40. Bjourson, A.J.; Cooper, J.E. Band-stab pcr: A simple technique for the purification of individual pcr products. Nucleic Acids Res. 1992, 20, 4675. [CrossRef]

41. Aung-Htut, M.T.; Comerford, I.; Johnsen, R.; Foyle, K.; Fletcher, S.; Wilton, S.D. Reduction of integrin alpha 4 activity through splice modulating antisense oligonucleotides. Sci. Rep. 2019, 9, 12994. [CrossRef]

(C) 2019 by the authors. Licensee MDPI, Basel, Switzerland. This article is an open access article distributed under the terms and conditions of the Creative Commons Attribution (CC BY) license (http://creativecommons.org/licenses/by/4.0/). 